\title{
Occupational exposure to fungi in gymnasiums with swimming pools
}

\author{
C. Viegas ${ }^{1}$, C. Alves ${ }^{2}$, E. Carolino ${ }^{1}$, L. Rosado ${ }^{2} \&$ C. Silva Santos ${ }^{3}$ \\ ${ }^{1}$ Higher School of Health Technologies of Lisbon, \\ Polytechnic Institute of Lisbon, Portugal \\ ${ }^{2}$ National Institute of Health Dr. Ricardo Jorge, \\ Mycology Laboratory, Portugal \\ ${ }^{3}$ School of Public Health, New University of Lisbon, Portugal
}

\begin{abstract}
Fungal contamination of the floor in 10 gyms with swimming pools was monitored. One hundred and twenty swab samples were collected: 60 before and 60 after cleaning operations. The samples were taken near the pool and jacuzzi, in surrounding the pool access stairs, in training studios and in male and female showers and changing rooms. Simultaneously, environmental parameters temperature and relative humidity - were also monitored. Thirty-seven different species of fungi were identified. Among those species, Fusarium sp. was the most frequent species isolated before $(19,1 \%)$ and after $(17,2 \%)$ the cleaning operations. Twelve different species of yeasts were identified. The most identified genera before cleaning was Cryptococcus $(40,6 \%)$, and after cleaning was Candida (49,3\%). The difference between before and after cleaning operations was statistically significant $(p<0,05)$ for fungi in pool access stairs and jacuzzi, and for yeasts in male showers and changing rooms. Taking into account the average values of fungal contamination the decreased due to cleaning procedures only occurred in male shower and changing rooms and only in yeasts count. The fungal contamination showed no significant relationship $(\mathrm{p}>0,05)$ with temperature and relative humidity.
\end{abstract}

Keywords: gymnasiums, swimming pools, fungal contamination, environmental variables, temperature, humidity. 


\section{Introduction}

Fungi presence requires ideal conditions of temperature, humidity, oxygen, carbon sources, nitrogen and minerals. Their biological activities of biodegradation and biodeterioration, depend on their enzymes activity, the environmental conditions, the competition phenomenon and the nature of the substrate [1].

According to the Achilles Project, developed in sixteen European countries in 2003, 34.9\% of 70497 subjects had feet fungal infections, being together Tinea pedis and onychomycosis the most common [1,2]. Tinea pedis, commonly known as "athlete foot", is an inflammatory condition and the most common form of dermatomycosis. It can be transmitted either by direct contact between two individuals or, indirectly, through contaminated objects or surfaces. Onychomycosis is usually associated with Tinea pedis and may occur due to trauma in the nail during physical activity. Both diseases can occur due exposure to contaminated surfaces with bare feet [3].

Regarding the etiological agents of these diseases, dermatophytes such as Trichophyton mentagrophytes, Trichophyton rubrum and Epidermophyton floccosum are the most commonly isolated in pools. For non-dermatophytic moulds (NDM) the most frequently identified in pools include the genera Alternaria, Aspergillus, Cladosporium, Fusarium, Geotrichum, Penicillum and Phoma [4]. Concerning yeasts, besides Candida albicans, species of Trichosporon sp. were also identified near the swimming pools [5].

Environmental variables such as temperature, humidity and substrate of the nutritional environment may enhance the fungal spread, contributing to the occurrence of Tinea pedis and onychomycosis. These may occur due to the exposure of workers and users of the gym with swimming pools. In this sense, it is necessary to know better the work conditions. However, until now, epidemiological studies have failed to establish a causal relationship between the extent of fungal presence and exposure time and specific effects on health or between frequency and severity of symptoms reported. Studies tend to show only the existence of a link between exposure to fungi and the development of symptoms [6].

For fungal contamination on surfaces, there is no Portuguese law setting a reference and for that it will be necessary to developed guidelines for professional and recreational settings.

This investigation was designed to describe the environmental fungal contamination phenomena in floor of gyms with swimming pools and explore possible associations with independent environmental variables.

\section{Materials and methods}

A descriptive study was developed to monitor the fungal floor contamination in the ten most popular gymnasiums in Lisbon. One hundred and twenty swab samples were collected: 60 before and 60 after the cleaning operations. The 
samples were taken near the pool and jacuzzi, in surrounding the pool access stairs, in training studios and in male and female showers and changing rooms.

Simultaneously, two environmental parameters - temperature and relative humidity - were monitored, using the Babouc equipment, (LSI Sistems), according to the International Standard ISO 7726 - 1998.

Swabs were performed according to the International Standard ISO 18593 2004 , using a $10 \mathrm{~cm}$ square of metal disinfected with $70 \%$ alcohol solution between samples. Swabs were inoculated in triplicate on malt extract agar with chloramphenicol as a bacteria growth inhibitor (MEA) and in mycobiotic agar with cycloheximide (MA). Subsequently, these were incubated at $27{ }^{\circ} \mathrm{C}$ (MEA for 5 to 7 days and MA for 15 to 20 days).

After laboratory processing and incubation of the collected samples, quantitative $\left(\mathrm{CFU} / \mathrm{m}^{2}\right)$ and qualitative results were obtained, with identification of isolated fungal species. Whenever possible, filamentous fungi and yeasts were identified to the species level, since adverse health effects vary according to fungal species [7, 8]. Identification of filamentous fungi was carried out on material mounted in lactophenol and achieved through morphological characteristics listed in illustrated literature [8] and yeasts were identified through biochemical API test [2].

With the obtained data, tables with frequency distribution of isolated fungal species were made. We compared the fungal contamination values before and after cleaning. Fungal concentration dependence in the two monitored environmental parameters - temperature and relative humidity - was also analyzed.

\section{Results}

Regarding fungi, thirty-seven species were isolated. Among these, the genera more frequent, before and after cleaning, were Fusarium (19,1\%-17,2\%), Penicillium (11,5\% - 16,9\%) and Scytalidium (11,5\% - 13,3\%).

Twenty-nine different species were identified before cleaning. Among Fusarium genus, the most frequent was Fusarium oxysporum and among Aspergillus genus, Aspergillus versicolor was the most commonly isolated. Concerning dermathophytes, $T$. mentagrophytes was the only species identified.

Twenty-five different species were identified after cleaning. Among Fusarium genus, Fusarium oxysporum was again the most frequent. Regarding dermathophytes, besides T. mentagrophytes, T. rubrum was also isolated.

Twelve different species of yeasts were identified. The most identified genera before cleaning was Cryptococcus $(40,6 \%)$, and after cleaning was the genera Candida (49,3\%). Before and after cleaning Trichosporon mucoides was the most frequent.

Ten different species of yeasts were identified before cleaning, namely Trichosporon mucoides, Rhodotorula sp., Candida sp., Candida parapsilosis, Candida guilliermondii, Cryptococcus humicola, Cryptococcus curvatus, Cryptococcus laurentii, Cryptococcus albidus and Cryptococcus uniguttulatus. 
Table 1: $\quad$ Most frequent fungi and yeasts identified in the surface of the 10 establishments monitored.

\begin{tabular}{|c|c|c|}
\hline Fungi & $\begin{array}{c}\text { Before cleaning } \\
\text { Frequency (\%) }\end{array}$ & $\begin{array}{c}\text { After cleaning } \\
\text { Frequency (\%) }\end{array}$ \\
\hline Fusarium sp. & 19,1 & 17,2 \\
Penicillium sp. & 11,5 & 16,9 \\
Scytalidium sp. & 11,5 & 13,3 \\
Phoma sp. & 10,7 & 10,3 \\
Cladosporium sp. & 8,4 & 3,3 \\
Aspergillus sp. & 6,1 & 4,2 \\
Tricophyton sp. & 2 & 1,1 \\
Others & 30,7 & 33,7 \\
\hline Yeasts & Before cleaning & After cleaning \\
\hline Cryptococcus sp. & Frequency (\%) & Frequency (\%) \\
\hline Candida sp. & 40,6 & 7,8 \\
Trichosporon sp. & 25,1 & 49,3 \\
Rhodotorula sp. & 21,7 & 37,1 \\
\hline
\end{tabular}

Eleven different species of yeasts were identified after cleaning, and besides Cryptococcus curvatus, those already identified before cleaning were also identified after cleaning. In addition, Candida famata and Cryptococcus neoformans were also isolated after cleaning (Table 1).

Regarding surfaces contamination, CFU were not isolated in 5 of the 60 monitored surfaces. In the remaining 55, $26(47,3 \%)$ presented more CFU $/ \mathrm{m}^{2}$ after cleaning and $23(41,8 \%)$ presented more $\mathrm{CFU} / \mathrm{m}^{2}$ before cleaning. Six $(10,9 \%)$ monitored surfaces didn't show any difference before and after cleaning.

Using the Wilcoxon rank test the difference between before and after cleaning was statistically significant $(\mathrm{p}<0,05)$ for fungi in pool access stairs and jacuzzi, and for yeasts in male showers and changing rooms. Moreover, taking into account the average values of fungal contamination, the decreased due to cleaning procedures only occurred in male shower and changing rooms and only in yeasts count.

From the results we can conclude that the values for $\mathrm{CFU} / \mathrm{m}^{2}$ were always higher for yeasts than for fungi, before and after cleaning.

Regarding the influence of environmental variables monitored no significant correlation $(p>0,05)$ was reveal. Temperature and relative humidity contributed only in $7,52 \%$ and $5,07 \%$, respectively, to $\mathrm{CFU} / \mathrm{m}^{2}$ variation explanation (Figures 1 and 2). 


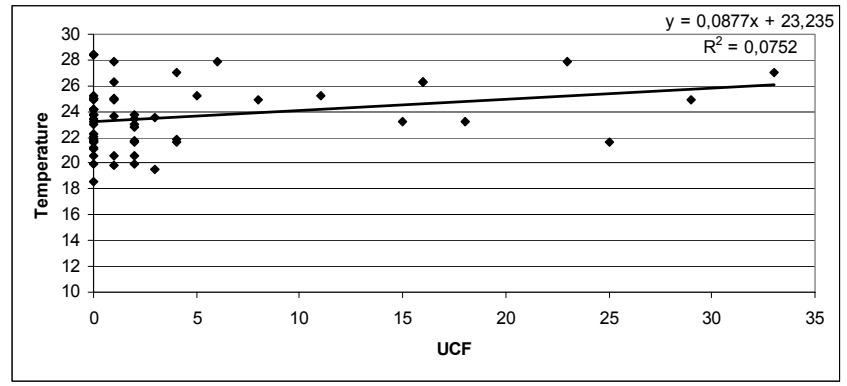

Figure 1: Influence of temperature in $\mathrm{CFU} / \mathrm{m}^{2}$.

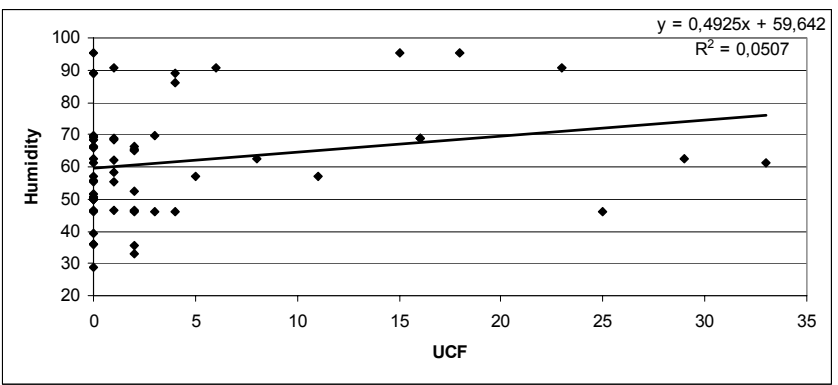

Figure 2: Influence of relative humidity in $\mathrm{CFU} / \mathrm{m}^{2}$.

\section{Discussion}

There is a wide diversity of clinical forms from Tinea pedis and onychomycosis and their etiologic agents may be dermatophytes, NDM and yeasts. Some of the species identified can be Tinea pedis and onychomycosis etiological agents, namely dermatophytes such as T. mentagrophytes and T. rubrum [4], NDM including the genera Fusarium, Scytalidium, Aspergillus, Cladosporium and Phoma [9] and yeasts belonging the genera Candida, Trichosporon and Cryptococcus $[10,11]$. Most authors diagnose as the most frequent etiological agents the dermatophytes ( 80 to $90 \%$ ), followed by yeasts ( 5 to $17 \%$ ) and finally $\operatorname{NDM}(2$ to $12 \%)$ [9].

Regarding dermatophytes, the species T. rubrum and T. mentagrophytes were isolated in 5 gyms with swimming pools and may have been spread by individuals with injury or asymptomatic individuals, due to the fact that dermatophytes can be isolate in individuals without injury [13] and there are anthropophilic species. Besides in 3 times this species were isolated was after cleaning, presumably due to the cleaning procedures and/or unsuitable products used for the elimination of some fungal species [14]. 
In a study that took place from 1980 to 2005 in the United Kingdom (UK), it was found that $T$. rubrum and T. mentagrophytes, comprised $80 \%$ of all dermatophytes isolated in 1980 and $90 \%$ of isolates in 2005 . In the same study, T. rubrum continues to be the most frequent isolated among all other fungal species isolated in the UK and is the causal agent of foot infection [15]. T. rubrum was also the most isolated in Portugal, in a study developed in Braga [16], involving 10003 patients, and where T. rubrum was identified in 882 cases (37.4\%). Therefore, identification of these 2 species in 5 out of 10 establishments was no surprise.

Concerning NDM, Fusarium sp. was also the most common, as in a study developed in Italian pools [5]. The 3 isolated species belonging to this genus were $F$. clamidosporo, $F$. oxysporum and $F$. solani, and the latter two are the most common cause of onychomycosis by NDM. In recent years, increased diagnose of onychomycosis caused by these fungi, particularly Fusarium sp., justify inclusion of these fungi as possible etiologic agents of Tinea pedis and onychomycosis $[2,10]$.

Like the dermatophytes Scytalidium sp., also isolated in the study, is considered a pathogen in tropical regions. Both genus, Scytalidium and Fusarium, are able to metabolize the keratin in nails, but with less intensity than dermatophytes [17].

Aspergillus sp. is often isolated in onychomycosis [9] and it was identified in surfaces before and after cleaning. Several species were identified, namely Aspergillus ochraceus, Aspergillus flavus, Aspergillus. fumigatus, Aspergillus nidulans, Aspergillus niger and Aspergillus versicolor.

Regarding yeasts, various infections have been noted due to $C$. albicans, $C$. famata, C. parapsilosis, C. guilliermondii, Cryptococcus albidus, Cryptococcus laurentii, Cryptococcus neoformans and Rhodotorula genus and all have been isolated in this study. The species of the Candida genus, the most common after cleaning, are opportunistic pathogens that appear to cause invasive infections in debilitated host [11].

Cryptococcus genus was the most common isolated yeast before cleaning and some of the isolated species are pathogenic. The species C. neoformans, isolated only after cleaning, is considered the leading cause of central nervous system fungal infection in immunocompromised individuals, and has been considered as a systemic pathogenic [18]. Incidence of Trichosporon genus has been increasing and is also related with infections in debilitated host [11]. Trichosporon mucoides was the most frequent yeast before and after cleaning.

In the 55 monitored places with fungal contamination, 26 had greater number of $\mathrm{CFU} / \mathrm{m}^{2}$ after cleaning. Furthermore, the differences were statistically significant between before and after cleaning for fungi $\left(\mathrm{CFU} / \mathrm{m}^{2}\right)$ on pool area access stairs and near the jacuzzi, and for yeasts $\left(\mathrm{CFU} / \mathrm{m}^{2}\right)$ in male showers and changing rooms. In addition, taking into account the average values of fungal contamination, decreased occurred after cleaning in male shower and changing rooms, and only in yeasts count. This situation may be due to inappropriate cleaning procedures, inadequate products and because cross-contamination can 
occur due the use of common materials. These justifications may also explain the fact that different fungal species appeared after cleaning.

It was found that before and after cleaning the CFU counts were higher for yeasts than for fungi, which may be due to the fact that yeasts are more difficult to disseminate in the air [7] or because they are more resistant to the products used on surfaces during the cleaning procedures.

Results related to environmental variables are not consistent with the expected [19]. It was found that the relationship between the fungal contamination and the temperature and relative humidity was not statistically significant $(p>0,05)$. This may be justified by the effect of other environmental variables also influencing fungal spreading, namely cleaning operations applied to monitored areas, components of heating, ventilation and air conditioning systems [20] and, also, users and professionals, who may carry, in their own body (commensal flora) or clothing, a great diversity of fungal species [21]. Besides this, activities as simple as walking in the monitored area, may also affect fungal concentration [22].

\section{Conclusions}

With the collected data it was possible to characterize fungal distribution in surfaces of different areas of gyms with swimming pools and to evaluate the association of environmental variables with this distribution. It was also possible to verify that several isolated fungal species can be responsible for Tinea pedis and onychomycosis.

Also relevant is that the decreased in fungal contamination due to cleaning procedures only occurred in male shower and changing rooms and only in yeasts count. Furthermore, after cleaning different fungal species appeared. Besides that, CFU count was higher in yeasts than in fungi.

Unlike other studies, environmental variables monitored (temperature and relative humidity) did not show the expected association with fungal concentration, which may possibly have resulted from other variables not investigated in this study.

\section{References}

[1] Burzykowski T, Molenberghs G, Abeck D, Haneke E, et al., High prevalence of foot diseases in Europe: results of the Achilles Project. Mycoses, 46, pp. 496 - 505, 2003.

[2] Ghannoum M, Hajeh R, Scher R, Konnikov N, et al., A large-scale North American study of fungal isolates from nails: The frequency of onychomycosis, fungal distribution and antifungal susceptibility patterns. $J$. Am. Acad. Dermatol, 43, pp. 641 - 648, 2000.

[3] Caputo R, De Boville K, Del Rosso J \& Nowicki R., Prevalence of superficial fungal infections among sports-active individuals: results from the Achilles Survey, a review of the literature. European Academy of Dermatology and Venereology, 15, pp. 312-216, 2001. 
[4] Ali-Shtayeh M, Khaleel T \& Jamous R., Ecology of dermatophytes and other keratinophilic fungi in swimming pools and polluted and unpolluted streams. Mycopathologia, 156, pp. 193-205, 2002.

[5] Brandi G, Sisti M, Paparini A, Gianfranceschi G, et al., Swimming pools and fungi: An environmental epidemiology survey in Italian indoor swimming facilities. International Journal of Environmental Health Research, 17, pp. 197 - 206, 2007.

[6] Goyer N, Lavoie J, Lazure L \& Marchand G. Bioaerosols in the Workplace: Evaluation, Control and Prevention Guide. Institut de Recherche en Santé et en Sécurité du Travail du Québec, 2001.

[7] Rao C, Burge H \& Chang J., Review of quantitative standards and guidelines for fungi in indoor air. J Air Waste Manage Assoc., 46, pp. 899 908, 1996.

[8] Hoog C, Guarro J, Gené G \& Figueiras M., (2 ${ }^{\text {nd }}$ ed). Atlas of Clinical Fungi. Centraalbureau voor Schimmelcultures, 2000.

[9] Gianni C, Cerri A \& Crosti C., Non-dermatophytic onychomycosis. An underestimated entity? A study of 51 cases. Mycoses, 43, pp. 29 - 33, 2000.

[10] Araújo A, Sousa M, Bastos O \& Oliveira J., Onicomicoses por fungos emergentes: análise clínica, diagnóstico laboratorial e revisão. An Bras Dermatol., 78, pp. 455-55, 2003.

[11] Odds F., ( $2^{\text {nd }}$ ed). Candida and Candidosis. Baillière Tindall. 1988.

[12] Kenna M \& Elewski B., A US epidemiological survey of superficial fungal diseases. J. Am. Acad Dermatol, 35, pp. 539 - 542, 1996.

[13] Attye A, Auger P \& Joly, J., Incidence of occult athletes's foot in swimmers. European Journal of Epidemiology, 3, pp. 244-247, 1990.

[14] Ferreira V \& Sousa J., Microbiologia. Lidel - Edições Técnicas, Lda. 2000.

[15] Borman A, Campbell C, Fraser M \& Johnson E., Analysis of the dermatophyte species isolated in the British Isles between 1980 and 2005 and review of worldwide dermatophyte trends over the last three decades. Medical Mycology, 45, pp. 131 - 141, 2007.

[16] Valdigem G, Pereira T, Macedo C, Duarte M, et al., A twenty-year survey of dermatophytoses in Braga, Portugal. Int J Dermatol., 45, pp. 822 - 7, 2006.

[17] Gupta A, Cooper E, Macdonald P \& Summerbell R., Utility of inoculum counting (Walshe and English criteria) in clinical diagnosis of onychomycosis caused by nondermatophitic filamentous fungi. $J$ Clin Microbiol., 9, pp. $2115-21,2001$.

[18] Kauffman C. \& Mandell G., (2 ${ }^{\text {nd }}$ ed). Atlas of Fungal Infections. Springer, 2007.

[19] Kakde U, Kakde H \& Saoji A., Seasonal Variation of Fungal Propagules in a Fruit Market Environment, Nagpur (India). Aerobiologia, 17, pp. 177 182, 2001.

[20] Kemp P, Neumeister-Kemp H, Esposito B, Lysek G \& Murray F., Changes in airborne fungi from the outdoors to indoor air; Large HVAC systems in nonproblem buildings in two different climates. American Industrial Hygiene Association, 64, pp. 269 - 275, 2003. 
[21] Scheff P, Pulius V, Curtis L \& Conroy L., Indoor air quality in a middle school, Part II: Development of emission factors for particulate matter and bioaerosols. Applied Occupational and Environmental Hygiene, 15, pp. 835 $-842,2000$.

[22] Buttner M \& Stetzenbach L., Monitoring Airborne fungal spores in an experimental indoor environment to evaluate sampling methods and the effects of human activity on air sampling. Applied and Environmental Microbiology, 59, pp. 219-226, 1993. 